\title{
New intermediates for the selective synthesis of 1-methyl-3- phenylpiperazine and some phenylpiperazine derivatives
}

\author{
Divvela V. N. Srinivasa Rao, ${ }^{a}$ Ramesh Dandala, ${ }^{a *}$ Vijay Kumar Handa, \\ Meenakshisunderam Sivakumaran, ${ }^{a}$ and Andra Naidu ${ }^{b}$ \\ ${ }^{a}$ Chemical Research Department, APL Research Centre, Hyderabad-500 072, India \\ ${ }^{b}$ J. N. T. University, Kukatpally, Hyderabad-500 072, Andhra pradesh, India \\ E-mail: rdandala@aurobindo.com
}

\begin{abstract}
New intermediates, 4-protected-1-alkyl-2-oxo-3-phenylpiperazines (3a-e) and 1-alkyl-2-oxo-3phenylpiperazines (6a-d) for the selective synthesis of 1-alkyl-3-phenylpiperazines (5a-e) are described. First method involves the reduction of the 4-protected-1-alkyl-2-oxo-3phenylpiperazine (3a-e) followed by deprotection giving the 1-alkyl-3-phenylpiperazine (5a-e). Second method involves the deprotection of 4-protected-1-alkyl-2-oxo-3-phenylpiperazine (3ae) followed by reduction giving the 1-alkyl-3-phenylpiperazine (5a-e).
\end{abstract}

Keywords: 3-Phenylpiperazin-2-one, 4-benzyl-1-methyl-2-oxo-3-phenylpiperazine, 1-methyl-2oxo-3-phenylpiperazine, protection, deprotection

\section{Introduction}

1-Methyl-3-phenylpiperazine is an intermediate for the preparation of Mirtazapine, an antidepressant drug. ${ }^{1}$ Several methods have been reported for the preparation of 1-methyl-3phenylpiperazine. They have been prepared from 2-phenylpiperazine, ${ }^{2} \mathrm{~N}$ - (2-chloroethyl)-Nmethyl- $\beta$-chloro- $\beta$-phenylethylamine, ${ }^{3} \quad$ 2-chloroacetamide-N-methyl-2-phenylacetamide, ${ }^{4} \quad 1$ benzyl-2-phenylpiperazine. ${ }^{5,6}$ Our present work describes new intermediates such as 4-benzyl-1methyl-2-oxo-3-phenylpiperazine ${ }^{7}$ and 1-methyl-2-oxo-3-phenylpiperazine ${ }^{7}$ for the preparation of 1-methyl-3-phenylpiperazine and some phenylpiperazine derivatives.

\section{Results and Discussion}

The method described by Roderick, ${ }^{2}$ involves the methylation of 2-phenylpiperazine and this step afforded low yields because it was not selective and produces unwanted product 1,4- 
dimethylpiperazine. By the route of Dolitzky, ${ }^{3}$ the piperazine was made from $N$ - (2-chloroethyl)$N$-methyl- $\beta$-chloro- $\beta$-phenylethylamine and this method afforded by product 1-methyl-2phenylpiperazine. This may be because of the non-selectivity in the reaction of starting material preparation. The method described by Guo, ${ }^{5}$ involves the use of excess of lithium aluminum hydride, ${ }^{7}$ for the unsubstituted amide reduction. We wish to report herein an improved procedure using new intermediates, which circumvents these problems. Different 1-alkyl-3phenylpiperazines were prepared in two methods by simply changing the sequence of reduction and deprotection of the phenylpiperazine derivatives. First method involves the reduction of 3a-e followed by the deprotection of 4a-e to get 1-alkyl-3-phenylpiperazine 5a-e (Scheme 1).

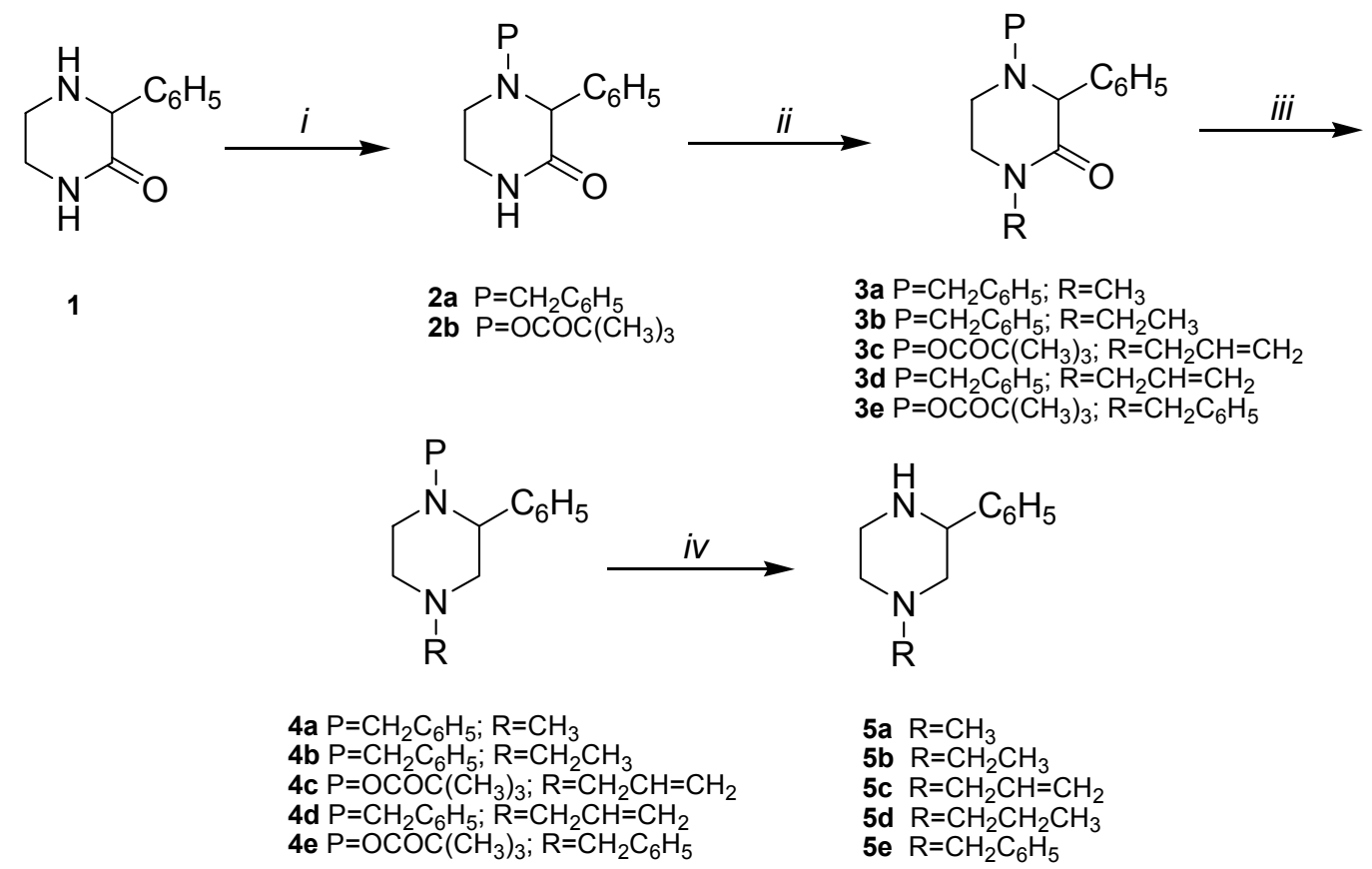

Scheme 1. $i$. Protection: for benzyl; $\mathrm{C}_{6} \mathrm{H}_{5} \mathrm{CH}_{2} \mathrm{Cl}, \mathrm{NaHCO}_{3}, \mathrm{DMF}, 100{ }^{\circ} \mathrm{C}$; for Boc. di-tert-butyl dicarbonate, $\left(\mathrm{C}_{2} \mathrm{H}_{5}\right)_{3} \mathrm{~N}, \mathrm{CH}_{2} \mathrm{Cl}_{2}$, rt. ii. $\mathrm{RI}, \mathrm{NaH}, \mathrm{DMF}, 25{ }^{\circ} \mathrm{C}$. iii. $\mathrm{LiAlH}_{4}, \mathrm{THF}$, reflux. $i v$. deprotection: for benzyl; $\mathrm{H}_{2}, \mathrm{Pd}-\mathrm{C}, \mathrm{CH}_{3} \mathrm{OH}, \mathrm{CH}_{3} \mathrm{COOH}$, rt; for Boc; $6 \mathrm{~N} \mathrm{HCl}$, rt.

Second method involves the deprotection of 3a-c, e followed by reduction of 1-alkyl-2oxo-3-phenylpiperazines 6a-d giving the 1-alkyl-3-phenylpiperazines 5a-c, e (Scheme 2). 


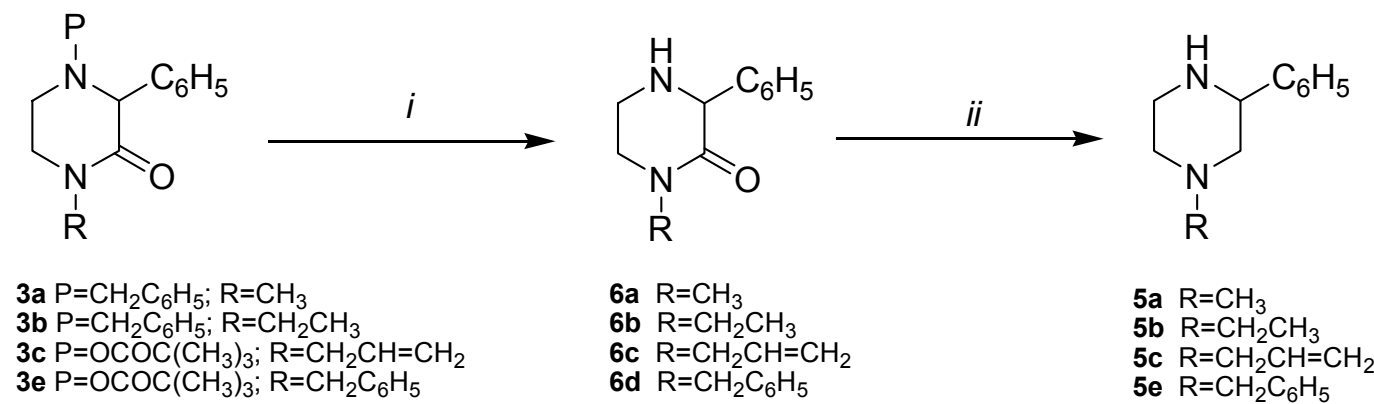

Scheme 2. $i$. deprotection: for benzyl; $\mathrm{H}_{2}$, Pd-C, acetic acid, rt. for Boc; $6 \mathrm{~N} \mathrm{HCl}, \mathrm{rt}, i i$. $\mathrm{LiAlH}_{4}$, THF, reflux.

In summary, we report two different sequences of reactions, viz. lactam reduction and $\mathrm{N}^{4}$ deprotection, or, initial $\mathrm{N}^{4}$-deprotection followed by lactam reduction, provided the title compounds in good overall yield. However, the intermediates (6a-d) obtained by following the second method may be useful for making some biological important compounds owing to the available free $\mathrm{NH}$ group.

\section{Experimental Section}

General Procedures. All melting points were determined with Palmon melting point apparatus. ${ }^{1} \mathrm{H}-\mathrm{NMR}$ spectra were recorded on a Bruker 300 spectrometer. Chemical shifts are reported in ppm downfield from TMS as internal standard. Mass spectra were measured on Perkin Elmer PE SCIEX-API 2000 mass spectrometer. Elemental analyses were performed using a Heraeus CHNO-Rapid instrument. Analytical HPLC ${ }^{8}$ were run with Hypersil ODS Rx-C 18 column at 210nm. "RT" denotes room temperature.

4-Benzyl-2-oxo-3-phenylpiperazine (2a). Benzyl chloride $(75.0 \mathrm{~g}, 0.593 \mathrm{~mol})$ was added to a suspension of 1 (100.0 g, $0.568 \mathrm{~mol}$ ) obtained by the reported method (lit. ${ }^{2} \mathrm{mp} 138-140{ }^{\circ} \mathrm{C}$ ) and sodium bicarbonate $(100.0 \mathrm{~g}, 1.19 \mathrm{~mol})$ in DMF $(250 \mathrm{~mL})$ and heated to $100{ }^{\circ} \mathrm{C}$ for $2 \mathrm{~h}$. Cooled the mixture to $75^{\circ} \mathrm{C}$ and water $(500 \mathrm{ml})$ was added. Filtered the product and washed with water, dried, to yield $2 \mathrm{a}\left(135.0 \mathrm{~g}, 89 \%\right.$ ) as a white solid; purity $99 \%$ (by HPLC); mp $225-226^{\circ} \mathrm{C}$ (lit, ${ }^{5}$ $\left.>210{ }^{\circ} \mathrm{C}\right)$; IR $\left(\mathrm{KBr}, \mathrm{cm}^{-1}\right) 3181,2891,1678,1602,742,698 ;{ }^{1} \mathrm{H}-\mathrm{NMR}\left(300 \mathrm{MHz}, \mathrm{CDCl}_{3}\right) \delta$ 2.48-2.54 (m, 1H), 2.98-3.00 (m, 1H), $3.17(\mathrm{~d}, 1 \mathrm{H}, J=13.5 \mathrm{~Hz}), 3.26-3.28(\mathrm{~m}, 1 \mathrm{H}), 3.52-3.44$ (m, 1H), 3.75 (d, 1H, J=13.5 Hz), 4.07 (s, 1H), 6.41 (bs, 1H), 7.20-7.30 (m, 5H), 7.32-7.41 (m, 3H), 7.54-7.55 (m, 2H); ${ }^{13} \mathrm{C}-\mathrm{NMR}\left(300 \mathrm{MHz}, \mathrm{CDCl}_{3}\right) \delta$ 46.1, 51.7, 63.7, 76.0, 132.3, 133.8, 132.8, 133.5, 134.3, 143.2, 144.6, 174.9; MS (ESI, $m / z): 267.4[\mathrm{M}+\mathrm{H}]^{+}$. Anal. Calcd. For $\mathrm{C}_{17} \mathrm{H}_{18} \mathrm{~N}_{2} \mathrm{O}$ (266.34): C, 76.66; H, 6.81; N, 10.52. Found: C, 76.70; H, 6.74; N, 10.45.

4-tert-Butoxycarbonyl-2-oxo-3-phenylpiperazine (2b). Di-tert-butyl dicarbonate (126.4 g, 0.58 mol) was added to a suspension $1(100.0 \mathrm{~g}, 0.568 \mathrm{~mol})$ and triethylamine $(72.72 \mathrm{~g}, 0.72 \mathrm{~mol})$ in dichloromethane $(1000 \mathrm{~mL})$ at $10^{\circ} \mathrm{C}$ and stirred at $\mathrm{RT}$ for $15 \mathrm{~h}$. The reaction mixture was washed 
with water and evaporated. The residue was treated with cyclohexane $(500 \mathrm{~mL})$. Filtered the product and washed with cyclohexane, dried, to yield $\mathbf{2 b}(125.0 \mathrm{~g}, 80 \%)$ as a white solid; mp 152-153 ${ }^{\circ} \mathrm{C}$; IR $\left(\mathrm{KBr}, \mathrm{cm}^{-1}\right)$ 3197, 2986, 1690, 1674, 1602, 1395, 737, 699; ${ }^{1} \mathrm{H}-\mathrm{NMR}$ (300 MHz, $\left.\mathrm{CDCl}_{3}\right) \delta 1.45(\mathrm{~s}, 9 \mathrm{H}), 3.27-3.34(\mathrm{~m}, 2 \mathrm{H}), 3.48-3.52(\mathrm{~m}, 1 \mathrm{H}), 3.92-4.04(\mathrm{~m}, 1 \mathrm{H}), 5.71(\mathrm{~s}, 1 \mathrm{H})$, 6.65 (bs, $1 \mathrm{H}), 7.28-7.44(\mathrm{~m}, 5 \mathrm{H}) ;{ }^{13} \mathrm{C}-\mathrm{NMR}\left(300 \mathrm{MHz}, \mathrm{CDCl}_{3}\right) \delta$ 28.8, 37.9, 41.1, 60.7, 81.4, 127.5, 128.3, 129.0, 137.9, 154.3, 170.1; MS (ESI, $m / z): 277.2[\mathrm{M}+\mathrm{H}]^{+}$. Anal. Calcd. For $\mathrm{C}_{15} \mathrm{H}_{20} \mathrm{~N}_{2} \mathrm{O}_{3}$ (276.33): C, 65.20; H, 7.30; N, 10.14. Found: C, 65.12; H, 7.34; N, 10.18 .

General procedure for alkylation of 4-protected-2-oxo-3-phenylpiperazines (2a-b). 4Benzyl-1-methyl-2-oxo-3-phenylpiperazine (3a). Under $\mathrm{N}_{2}$ atmosphere, 2a (100.0 g, 0.376 mol) was added portion wise to a suspension of sodium hydride (15.3 g, 65\% dispersed in mineral oil, $0.41 \mathrm{~mol})$ in anhydrous DMF $(250 \mathrm{~mL})$ at $10-15^{\circ} \mathrm{C}$ over a period of $1 \mathrm{~h}$. A solution of methyl iodide $(64.0 \mathrm{~g}, 0.45 \mathrm{~mol})$ in anhydrous DMF $(65 \mathrm{~mL})$ was added slowly by maintaining the temperature below $25^{\circ} \mathrm{C}$ and the stirring was continued for $1 \mathrm{~h}$. The reaction mass was poured slowly into cold water $(1000 \mathrm{~mL})$ and product was extracted with toluene $(800 \mathrm{~mL})$, the combined organic solution was washed with water and concentrated. The residue was treated with cyclohexane $(200 \mathrm{~mL})$ at $10{ }^{\circ} \mathrm{C}$ for $1 \mathrm{~h}$. Filtered the product to yield $\mathbf{3 a}(98.5 \mathrm{~g}$, 94\%) as a light yellow solid; purity $99 \%$ (by HPLC); mp 101-102 ${ }^{\circ} \mathrm{C}$; IR ( $\left.\mathrm{KBr}, \mathrm{cm}^{-1}\right) 2935$, 2805, 1646, 1602, 733, 697; ${ }^{1} \mathrm{H}-\mathrm{NMR}\left(300 \mathrm{MHz}, \mathrm{CDCl}_{3}\right) \delta$ 2.54-2.58 (m, 1H), 2.99 (s, 3H), 3.03-3.05 (m, 1H), $3.17(\mathrm{~d}, 1 \mathrm{H}, J=13.5 \mathrm{~Hz}), 3.15-3.20(\mathrm{~m}, 1 \mathrm{H}), 3.22-3.60(\mathrm{~m}, 1 \mathrm{H}), 3.75(\mathrm{~d}, 1 \mathrm{H}$, $J=13.4 \mathrm{~Hz}), 4.08(\mathrm{~s}, 1 \mathrm{H}), 7.26-7.32(\mathrm{~m}, 6 \mathrm{H}), 7.35-7.40(\mathrm{~m}, 2 \mathrm{H}), 7.55(\mathrm{~d}, 2 \mathrm{H}, J=7.4 \mathrm{~Hz}) ;{ }^{13} \mathrm{C}-$ NMR $\left(300 \mathrm{MHz}, \mathrm{CDCl}_{3}\right) \delta 35.1,46.9,48.9,59.3,71.5,127.7,128.2,128.8,129.2,129.5,138.4$, 139.7, 168.7; MS (ESI, $m / z): 281.2[\mathrm{M}+\mathrm{H}]^{+}$. Anal. Calcd. For $\mathrm{C}_{18} \mathrm{H}_{20} \mathrm{~N}_{2} \mathrm{O}$ (280.36): C, 77.11; H, 7.19; N, 9.99. Found: C, 77.03; H, 7.22; N, 9.96.

4-Benzyl-1-ethyl-2-oxo-3-phenylpiperazine (3b). This compound was prepared in a similar way to 3a, using compound $\mathbf{2 a}(8.0 \mathrm{~g}, 30.07 \mathrm{mmol})$ and ethyl bromide, as a light yellow solid (8.13 g, 92\%); mp 88-89 ${ }^{\circ} \mathrm{C}$; IR $\left(\mathrm{KBr}, \mathrm{cm}^{-1}\right)$ 2937, 2818, 1648, 1602, 742, 699; ${ }^{1} \mathrm{H}-\mathrm{NMR}$ (300 $\left.\mathrm{MHz}, \mathrm{CDCl}_{3}\right) \delta 1.13(\mathrm{t}, 3 \mathrm{H}, J=7.2 \mathrm{~Hz}), 2.50-2.54(\mathrm{~m}, 1 \mathrm{H}), 3.03-3.13(\mathrm{~m}, 1 \mathrm{H}), 3.15-3.18(\mathrm{~m}, 2 \mathrm{H})$, 3.34-3.38 (m, 1H), 3.46-3.53 (m, 2H), 3.72-3.77 (d, 1H, J=13.4 Hz), $4.04(\mathrm{~s}, 1 \mathrm{H}), 7.21-7.38(\mathrm{~m}$, $8 \mathrm{H}), 7.52(\mathrm{~d}, 2 \mathrm{H}, J=7.4 \mathrm{~Hz}) ;{ }^{13} \mathrm{C}-\mathrm{NMR}\left(300 \mathrm{MHz}, \mathrm{CDCl}_{3}\right) \delta 12.1,41.8,45.7,46.6,58.8,71.0$, 127.2, 127.7, 128.3, 128.7, 129.0, 137.9, 139.5, 167.6; MS (ESI, $m / z): 295.3[\mathrm{M}+\mathrm{H}]^{+}$. Anal. Calcd. For $\mathrm{C}_{19} \mathrm{H}_{22} \mathrm{~N}_{2} \mathrm{O}$ (294.39): C, 77.52; H, 7.53; N, 9.52. Found: C, 77.46; H, 7.50; N, 9.56.

1-Allyl-4-tert-butoxycarbonyl -2-oxo-3-phenylpiperazine (3c). This compound was prepared in a similar way to $\mathbf{3 a}$, using compound $\mathbf{2 b}(8.0 \mathrm{~g}, 28.98 \mathrm{mmol})$ and allyl bromide, as a light yellow liquid (8.2 g, 92\%); IR (neat, $\mathrm{cm}^{-1}$ ) 3083, 1698, 1658, 1392, 721, 698; ${ }^{1} \mathrm{H}-\mathrm{NMR}(300$ $\left.\mathrm{MHz} \mathrm{CDCl}_{3}\right) \delta 1.44(\mathrm{~s}, 9 \mathrm{H}), 3.23-3.28(\mathrm{~m}, 2 \mathrm{H}), 3.32-3.36(\mathrm{~m}, 1 \mathrm{H}), 3.85-3.97(\mathrm{~m}, 2 \mathrm{H}), 4.19-4.21$ $(\mathrm{m}, 1 \mathrm{H}), 5.15-5.21(\mathrm{~m}, 2 \mathrm{H}), 5.71-5.79(\mathrm{~m}, 2 \mathrm{H}), 7.29-7.38(\mathrm{~m}, 5 \mathrm{H}) ;{ }^{13} \mathrm{C}-\mathrm{NMR}\left(300 \mathrm{MHz}, \mathrm{CDCl}_{3}\right)$ $\delta 28.1,37.7,44.8,49.0,60.6,80.7,118.0,125.2,126.6,127.5,127.9,128.3,133.1,137.4,153.7$, 166.3; MS (ESI, $m / z): 317.3[\mathrm{M}+\mathrm{H}]^{+}$. Anal. Calcd. For $\mathrm{C}_{18} \mathrm{H}_{24} \mathrm{~N}_{2} \mathrm{O}_{3}$ (316.39): C, 68.33; H, 7.65; N, 8.85. Found: C, 68.40; H, 7.58; N, 8.82. 
1-Allyl-4-benzyl-2-oxo-3-phenylpiperazine (3d). This compound was prepared in a similar way to 3a, using compound 2a $(8.0 \mathrm{~g}, 30.07 \mathrm{mmol})$ and allyl bromide, as a yellow oil $(8.37 \mathrm{~g}, 91 \%)$; IR (neat, $\mathrm{cm}^{-1}$ ) 3084, 2804, 1649, 1603, 754, 700; ${ }^{1} \mathrm{H}-\mathrm{NMR}\left(300 \mathrm{MHz}, \mathrm{CDCl}_{3}\right) \delta 2.51-2.53(\mathrm{~m}$, $1 \mathrm{H}), 3.01-3.14(\mathrm{~m}, 1 \mathrm{H}), 3.16-3.20(\mathrm{~m}, 2 \mathrm{H}), 3.48-3.52(\mathrm{~m}, 1 \mathrm{H}), 3.75-3.80(\mathrm{~d}, 1 \mathrm{H}, J=13.4 \mathrm{~Hz})$, 3.98-4.05 (m, 2H), $4.10(\mathrm{~s}, 1 \mathrm{H}), 5.17-5.23(\mathrm{~m}, 2 \mathrm{H}), 5.71-5.84(\mathrm{~m}, 1 \mathrm{H}), 7.28-7.41(\mathrm{~m}, 8 \mathrm{H})$, 7.57(m, 2H); ${ }^{13} \mathrm{C}-\mathrm{NMR}\left(300 \mathrm{MHz}, \mathrm{CDCl}_{3}\right) \delta 45.8,46.6,49.3,58.8,71.0,117.8,127.2,127.8$, 128.3, 128.7, 129.0, 132.3, 137.8, 139.2, 162.5, 168.0; MS (ESI, $m / z): 307.5[\mathrm{M}+\mathrm{H}]^{+}$. Anal. Calcd. For $\mathrm{C}_{20} \mathrm{H}_{22} \mathrm{~N}_{2} \mathrm{O}$ (306.40): C, 78.40; H, 7.24; N, 9.14. Found: C, 78.51; H, 7.22; N, 9.10.

1-Benzyl-4-tert-butoxycarbonyl-2-oxo-3-phenylpiperazine (3e). This compound was prepared in a similar way to $\mathbf{3 a}$, using compound $\mathbf{2 b}(8.0 \mathrm{~g}, 28.98 \mathrm{mmol})$ and benzyl bromide, as a light brown oil (9.75 g, 92\%); IR (neat, $\mathrm{cm}^{-1}$ ) 3062, 2877, 1676, 1603, 1389, 729, 702; ${ }^{1} \mathrm{H}-\mathrm{NMR}(300$ $\left.\mathrm{MHz} \mathrm{CDCl}_{3}\right) \delta 1.45(\mathrm{~s}, 9 \mathrm{H}), 3.20-3.23(\mathrm{~m}, 1 \mathrm{H}), 3.32-3.36(\mathrm{~m}, 2 \mathrm{H}), 3.93(\mathrm{~m}, 1 \mathrm{H}), 4.49(\mathrm{~d}, 1 \mathrm{H}$, $J=14.5 \mathrm{~Hz}), 4.85(\mathrm{~d}, 1 \mathrm{H}, J=14.5 \mathrm{~Hz}), 5.78(\mathrm{~s}, 1 \mathrm{H}), 7.27-7.41(\mathrm{~m}, 10 \mathrm{H}) ;{ }^{13} \mathrm{C}-\mathrm{NMR}(300 \mathrm{MHz}$, $\left.\mathrm{CDCl}_{3}\right) \delta 28.6,38.8,45.3,50.4,60.9,81.3,127.9,128.1,128.4,128.6,128.7,128.9,129.1$, 129.4, 129.5, 138.1, 154.3, 162.8, 167.2; MS (ESI, m/z): $367.2[\mathrm{M}+\mathrm{H}]^{+}$. Anal. Calcd. For $\mathrm{C}_{22} \mathrm{H}_{26} \mathrm{~N}_{2} \mathrm{O}_{3}$ (366.45): C, 72.11; H, 7.15; N, 7.64. Found: C, 72.18; H, 7.18; N, 7.61.

General procedure for reduction of 4-protected-1-alkyl-3-phenylpiperazines (3a-e). 4Benzyl-1-methyl-3-phenylpiperazine (4a). Under $\mathrm{N}_{2}$ atmosphere, 3a (90.0 g, $0.321 \mathrm{~mol}$ ) was added slowly to a suspension of $\mathrm{LiAlH}_{4}{ }^{9}(14.6 \mathrm{~g}, 0.384 \mathrm{~mol})$ in anhydrous THF $(450 \mathrm{~mL})$ at 10$15{ }^{\circ} \mathrm{C}$ over a period of $1 \mathrm{~h}$ and refluxed for $6 \mathrm{~h}$. The excess $\mathrm{LiAlH}_{4}$ was destroyed at $0{ }^{\circ} \mathrm{C}$ with water $(14.6 \mathrm{~mL}), 15 \% \mathrm{NaOH}(14.6 \mathrm{~mL})$ and water $(42.8 \mathrm{~mL})$. After filtration, the filtrate was evaporated to dryness and water $(300 \mathrm{~mL})$ was added. Filtered the product to yield $\mathbf{4 a}(80.0 \mathrm{~g}$, 94\%) as a light yellow solid; purity $98 \%$ (by HPLC); mp 79-80 ${ }^{\circ} \mathrm{C}$; IR (KBr, cm ${ }^{-1}$ ) 2932, 2788, 1599, 740, 698; ${ }^{1} \mathrm{H}-\mathrm{NMR}\left(300 \mathrm{MHz}, \mathrm{CDCl}_{3}\right) \delta$ 2.15-2.22 (m, 2H), 2.23-2.28 (m, 1H), $2.99(\mathrm{~s}$, $3 \mathrm{H}), 2.80-2.85(\mathrm{~m}, 2 \mathrm{H}), 2.86(\mathrm{~d}, 2 \mathrm{H}, J=13.4 \mathrm{~Hz}), 3.44(\mathrm{dd}, 1 \mathrm{H}, J=10.5,2.9 \mathrm{~Hz}), 3.82(\mathrm{~d}, 1 \mathrm{H}$, $J=13.4 \mathrm{~Hz}), 7.19-7.30(\mathrm{~m}, 6 \mathrm{H}), 7.40-7.38(\mathrm{~m}, 2 \mathrm{H}), 7.50-7.52(\mathrm{~m}, 2 \mathrm{H}) ;{ }^{13} \mathrm{C}-\mathrm{NMR}(300 \mathrm{MHz}$, $\left.\mathrm{CDCl}_{3}\right) \delta 46.3,52.3,55.7,59.4,64.6,67.9,127.2,127.9,128.4,128.5,129.0,129.2,139.5$, 142.5; MS (ESI, $m / z)$ : $267.4[\mathrm{M}+\mathrm{H}]^{+}$. Anal. Calcd. For $\mathrm{C}_{18} \mathrm{H}_{22} \mathrm{~N}_{2}$ (266.38): C, 81.16; H, 8.32; N, 10.52. Found: C, 81.02; H, 8.44; N, 10.54 .

4-Benzyl-1-ethyl-3-phenylpiperazine (4b). This compound was prepared in a similar way to 4a, using compound $\mathbf{3 b}$ (5.0 g, $17.0 \mathrm{mmol}$ ), as a light yellow oil (4.52 g, 95\%); IR (neat, $\mathrm{cm}^{-1}$ ) 2945, 2805, 1603, 1470, 736, 700; ${ }^{1} \mathrm{H}-\mathrm{NMR}\left(300 \mathrm{MHz}, \mathrm{CDCl}_{3}\right) \delta 1.06$ (t, 3H, J=7.2 Hz), 2.05$2.19(\mathrm{~m}, 2 \mathrm{H}), 2.23-2.31(\mathrm{~m}, 1 \mathrm{H}), 2.36-2.44(\mathrm{~m}, 2 \mathrm{H}), 2.84-2.92(\mathrm{~m}, 4 \mathrm{H}), 3.41-3.44(\mathrm{~m}, 1 \mathrm{H}), 3.78$ $(\mathrm{d}, 1 \mathrm{H}, J=13.4 \mathrm{~Hz}), 7.19-7.37$ (m, 8H), 7.49-7.51 (m, 2H); ${ }^{13} \mathrm{C}-\mathrm{NMR}\left(300 \mathrm{MHz}, \mathrm{CDCl}_{3}\right) \delta 12.4$, 52.3, 52.6, 53.3, 59.5, 62.4, 67.8, 127.1, 127.9, 128.4, 128.5, 128.8, 129.0, 129.2, 139.5, 142.7; MS (ESI, $m / z): 281.3[\mathrm{M}+\mathrm{H}]^{+}$. Anal. Calcd. For $\mathrm{C}_{19} \mathrm{H}_{24} \mathrm{~N}_{2}$ (280.41): C, 81.38; H, 8.63; N, 9.99. Found: C, 81.24; H, 8.62; N, 10.14 .

1-Allyl-4-tert-butoxycarbonyl-3-phenylpiperazine (4c). This compound was prepared in a similar way to $\mathbf{4 a}$, using compound $\mathbf{3 c}(5.0 \mathrm{~g}, 15.82 \mathrm{mmol})$, as a light yellow oil $(3.72 \mathrm{~g}, 78 \%)$ after purification by silica gel (100-200 mesh) column chromatography [AcOEt-hexane (1:9)]; 
IR (neat, $\left.\mathrm{cm}^{-1}\right)$ 3063, 1694, 1603, 1392, 1300, 722, 700; ${ }^{1} \mathrm{H}-\mathrm{NMR}\left(300 \mathrm{MHz}, \mathrm{CDCl}_{3}\right) \delta 1.48(\mathrm{~s}$, $9 \mathrm{H}), 2.13-2.14(\mathrm{~m}, 1 \mathrm{H}), 2.31-2.37(\mathrm{~m}, 1 \mathrm{H}), 2.79-2.83(\mathrm{~m}, 2 \mathrm{H}), 3.03-3.08(\mathrm{~m}, 2 \mathrm{H}), 3.39-3.43(\mathrm{~m}$, $1 \mathrm{H}), 3.92-3.96(\mathrm{~m}, 1 \mathrm{H}), 5.17-5.26(\mathrm{~m}, 3 \mathrm{H}), 5.86-5.88(\mathrm{~m}, 1 \mathrm{H}), 7.24-7.43(\mathrm{~m}, 3 \mathrm{H}), 7.46(\mathrm{~m}, 2 \mathrm{H})$; ${ }^{13} \mathrm{C}-\mathrm{NMR}\left(300 \mathrm{MHz}, \mathrm{CDCl}_{3}\right) \delta 28.8,40.4,53.7,53.8,55.2,62.2,80.3,118.6,127.2,127.8$, 128.7, 135.2, 140.8, 155.4; MS (ESI, $m / z)$ : $303.3[\mathrm{M}+\mathrm{H}]^{+}$. Anal. Calcd. For $\mathrm{C}_{18} \mathrm{H}_{26} \mathrm{~N}_{2} \mathrm{O}_{2}$ (302.41): C, 71.49; H, 8.67; N, 9.26. Found: C, 71.28; H, 8.79; N, 9.29.

1-Allyl-4-benzyl -3-phenylpiperazine (4d). This compound was prepared in a similar way to 4a, using compound $\mathbf{3 d}(5.0 \mathrm{~g}, 16.33 \mathrm{mmol})$, as a light yellow solid (4.20 g, 88\%); mp 86-88 ${ }^{\circ} \mathrm{C}$. IR $\left(\mathrm{KBr}, \mathrm{cm}^{-1}\right)$ 3062, 2795, 1603, 741, 701; ${ }^{1} \mathrm{H}-\mathrm{NMR}\left(300 \mathrm{MHz}, \mathrm{CDCl}_{3}\right) \delta 2.07-2.14(\mathrm{~m}, 1 \mathrm{H})$, 2.18-2.30 (m, 2H), 2.81-2.90 (m, 4H), 2.97-2.99 (m, 2H), $3.40(\mathrm{dd}, 1 \mathrm{H}, J=10.4,2.7 \mathrm{~Hz}), 3.79$ $(\mathrm{dd}, 1 \mathrm{H}, J=13.4 \mathrm{~Hz}), 5.10-5.16(\mathrm{~m}, 2 \mathrm{H}), 5.73-5.84(\mathrm{~m}, 1 \mathrm{H}), 7.21-7.36(\mathrm{~m}, 8 \mathrm{H}), 7.49(\mathrm{~d}, 2 \mathrm{H}$, $\mathrm{J}=7.4 \mathrm{~Hz}) ;{ }^{13} \mathrm{C}-\mathrm{NMR}\left(300 \mathrm{MHz}, \mathrm{CDCl}_{3}\right) \delta 52.2,53.6,59.4,62.1,62.4,67.8,118.7,127.2,127.9$, $128.4,128.5,128.8,129.0,129.2,135.1,139.4,142.6$; MS (ESI, $m / z): 293.1[\mathrm{M}+\mathrm{H}]^{+}$. Anal. Calcd. For $\mathrm{C}_{20} \mathrm{H}_{24} \mathrm{~N}_{2}$ (292.42): C, 82.15; H, 8.27; N, 9.58. Found: C, 82.04; H, 8.30; N, 9.66.

1-Benzyl-4-tert-butoxycarbonyl -3-phenylpiperazine (4e). This compound was prepared in a similar way to $\mathbf{4 a}$, using compound $\mathbf{3 e}(5.0 \mathrm{~g}, 13.66 \mathrm{mmol})$, as a light yellow oil $(3.82 \mathrm{~g}, 79 \%)$ after purification by silica gel (100-200 mesh) column chromatography [AcOEt-hexane (1:9)]; IR (neat, $\left.\mathrm{cm}^{-1}\right)$ 2809, 1682, 1602, 1392, 737, 698; ${ }^{1} \mathrm{H}-\mathrm{NMR}\left(300 \mathrm{MHz}, \mathrm{CDCl}_{3}\right) \delta 1.47(\mathrm{~s}, 9 \mathrm{H})$, 2.15-2.19 (m, 1H), 2.38-2.43 (m, 1H), 2.78-2.82 (m, 1H), 2.99-3.06 (m, 1H), 3.26-3.30 (m, 1H), $3.44(\mathrm{~d}, 1 \mathrm{H}, J=13.2 \mathrm{~Hz}), 3.57$ (d, 1H, $J=13.2 \mathrm{~Hz}), 3.88-3.93(\mathrm{~m}, 1 \mathrm{H}), 5.23(\mathrm{~s}, 1 \mathrm{H}), 7.21-7.36$ (m, $8 \mathrm{H}), 7.41(\mathrm{~d}, 2 \mathrm{H}, J=7.4 \mathrm{~Hz}) ;{ }^{13} \mathrm{C}-\mathrm{NMR}\left(300 \mathrm{MHz}, \mathrm{CDCl}_{3}\right) \delta 28.9,31.7,40.3,53.8,55.4,63.6$, 80.3, 127.2, 127.6, 128.1, 128.5, 128.7, 129.6, 138.3, 140.9, 155.4; MS (ESI, m/z): 353.3 $[\mathrm{M}+\mathrm{H}]^{+}$. Anal. Calcd. For $\mathrm{C}_{22} \mathrm{H}_{28} \mathrm{~N}_{2} \mathrm{O}_{2}$ (352.47): C, 74.97; H, 8.01; N, 7.95. Found: C, 74.80; H, $8.15 ; \mathrm{N}, 7.98$.

1-Methyl-3-phenylpiperazine (5a). The mixture of 4a (60.0 g, $0.226 \mathrm{~mol}), 5 \% \mathrm{Pd}-\mathrm{C}(3.0 \mathrm{~g}$, $50 \%$ wet $),{ }^{10}$ methanol $(300 \mathrm{~mL})$ and acetic acid $(50 \mathrm{~mL})$ was hydrogenated with $\mathrm{H}_{2}(100 \mathrm{psi}$. $)$ at 25-30 ${ }^{\circ} \mathrm{C}$ for $5 \mathrm{~h}$, filtered and the filtrate was evaporated to dryness. Dissolved the residue in water $(150 \mathrm{~mL}), \mathrm{pH}$ adjusted to 11.5 with $40 \% \mathrm{NaOH}$ and extracted with toluene $(240 \mathrm{~mL})$. Combined organic solution was concentrated to dryness and the product was precipitated with cyclohexane. Filtered to yield 5a (36.2 g, 91\%) as a light yellow solid; purity 99.9\% (by HPLC); mp 58-60 ${ }^{\circ} \mathrm{C}$ (lit, $\left.{ }^{6} 53-55^{\circ} \mathrm{C}\right)$; IR $\left(\mathrm{KBr}, \mathrm{cm}^{-1}\right)$ 3253, 2942, 2816, 2792, 1603, 761, 703; ${ }^{1} \mathrm{H}-\mathrm{NMR}$ $\left(300 \mathrm{MHz}, \mathrm{CDCl}_{3}\right) \delta 1.76(\mathrm{bs}, 1 \mathrm{H}), 1.92-1.99(\mathrm{~m}, 1 \mathrm{H}), 2.10-2.15(\mathrm{~m}, 1 \mathrm{H}), 2.31(\mathrm{~s}, 3 \mathrm{H}), 2.80-$ $2.89(\mathrm{~m}, 2 \mathrm{H}), 3.01-3.11(\mathrm{~m}, 2 \mathrm{H}), 3.87(\mathrm{dd}, 1 \mathrm{H}, J=10.6,2.7 \mathrm{~Hz}), 7.27-7.41(\mathrm{~m}, 5 \mathrm{H}) ;{ }^{13} \mathrm{C}-\mathrm{NMR}$ $\left(300 \mathrm{MHz}, \mathrm{CDCl}_{3}\right) \delta 46.6,46.7,55.6,60.8,63.7,127.3,127.9,128.8,142.9 ; \mathrm{MS}(\mathrm{ESI}, \mathrm{m} / z)$ : $177.0[\mathrm{M}+\mathrm{H}]^{+}$. Anal. Calcd. For $\mathrm{C}_{11} \mathrm{H}_{16} \mathrm{~N}_{2}$ (176.26): C, 74.96; H, 9.15; N, 15.89. Found: C, 74.90; H, 9.22; N, 15.88 .

1-Ethyl-3-phenylpiperazine (5b). This compound was prepared in a similar way to $\mathbf{5 a}$, using compound 4b (3.0 g, $10.71 \mathrm{mmol}$ ), as a light yellow liquid (1.83 g, 90\%); IR (neat, $\mathrm{cm}^{-1}$ ) 3268, 2944, 2809, 1603, 1470, 756, 700; ${ }^{1} \mathrm{H}-\mathrm{NMR}\left(300 \mathrm{MHz}, \mathrm{CDCl}_{3}\right) \delta 1.10$ (t, 3H, J=7.2 Hz), $1.95-$ $1.98(\mathrm{~m}, 1 \mathrm{H}), 2.02-2.13(\mathrm{~m}, 1 \mathrm{H}), 2.45(\mathrm{q}, 2 \mathrm{H}, J=7.2 \mathrm{~Hz}), 2.91-2.97(\mathrm{~m}, 2 \mathrm{H}), 3.06-3.10(\mathrm{~m}, 2 \mathrm{H})$, 
3.87-3.91 (dd, $1 \mathrm{H}, J=10.4,2.7 \mathrm{~Hz}), 7.20-7.32(\mathrm{~m}, 3 \mathrm{H}), 7.34-7.41(\mathrm{~m}, 2 \mathrm{H}) ;{ }^{13} \mathrm{C}-\mathrm{NMR}(300 \mathrm{MHz}$, $\left.\mathrm{CDCl}_{3}\right) \delta 12.2,46.5,52.9,53.1,60.7,61.4,127.4,127.8,128.0,128.6,128.7,128.9,142.9 ; \mathrm{MS}$ (ESI, $m / z$ ): $191.2[\mathrm{M}+\mathrm{H}]^{+}$. Anal. Calcd. For $\mathrm{C}_{12} \mathrm{H}_{18} \mathrm{~N}_{2}$ (190.28): C, 75.74; H, 9.53; N, 14.72 . Found: C, 75.60; H, 9.61; N, 14.79 .

1-Allyl-3-phenylpiperazine (5c). Compound $4 \mathrm{c}(3.0 \mathrm{~g}, 9.93 \mathrm{mmol})$ was treated with $6 \mathrm{~N} \mathrm{HCl}(12$ $\mathrm{mL}$ ) at $25-30^{\circ} \mathrm{C}$ for $4 \mathrm{~h}$. Adjusted the $\mathrm{pH}$ to 11.5 with $40 \% \mathrm{NaOH}$ and the aqueous solution was extracted with dichloromethane $(30 \mathrm{~mL})$, evaporated to dryness to yield $\mathbf{5 c}$ as a light yellow liquid (1.76 g, 88\%); IR (neat, $\mathrm{cm}^{-1}$ ) 3069, 2886, 1603, 1471, 761, 698; ${ }^{1} \mathrm{H}-\mathrm{NMR}(300 \mathrm{MHz}$, $\left.\mathrm{CDCl}_{3}\right) \delta 1.98(\mathrm{br}, 1 \mathrm{H}), 3.56-3.68(\mathrm{~m}, 6 \mathrm{H}), 3.72-3.84(\mathrm{~m}, 2 \mathrm{H}), 4.85-4.88(\mathrm{~m}, 1 \mathrm{H})$, 5.51-5.63 (m, $2 \mathrm{H})$, 5.95-6.09 (m, 1H), 7.49-7.54 (m, 3H), 7.69-7.71(m, $2 \mathrm{H}) ;{ }^{13} \mathrm{C}-\mathrm{NMR}\left(300 \mathrm{MHz}, \mathrm{CDCl}_{3}\right) \delta$ 41.8, 47.0, 52.5, 57.1, 59.7, 125.6, 127.4, 128.7, 129.8, 130.8, 132.0; MS (ESI, $m / z): 203.3$ $[\mathrm{M}+\mathrm{H}]^{+}$. Anal. Calcd. For $\mathrm{C}_{13} \mathrm{H}_{18} \mathrm{~N}_{2}$ (202.30): C, 77.18; H, 8.97; N, 13.85. Found: C, 77.31; H, $8.88 ; \mathrm{N}, 13.81$.

1-Propyl-3-phenylpiperazine (5d). This compound was prepared in a similar way to 5a, using compound 4d (3.0 g, $10.27 \mathrm{mmol})$, as a light yellow liquid (1.92 g, 92\%); IR (neat, $\left.\mathrm{cm}^{-1}\right) 3257$, 2935, 2807, 1603, 755, 700; ${ }^{1} \mathrm{H}-\mathrm{NMR}\left(300 \mathrm{MHz}, \mathrm{CDCl}_{3}\right) \delta 0.90(\mathrm{t}, 3 \mathrm{H}, J=7.2 \mathrm{~Hz}), 1.50-1.53(\mathrm{~m}$, $2 \mathrm{H}), 1.95-2.11(\mathrm{~m}, 2 \mathrm{H}), 2.29-2.34(\mathrm{~m}, 2 \mathrm{H}), 2.85-2.93(\mathrm{~m}, 2 \mathrm{H}) 3.04-3.09(\mathrm{~m}, 2 \mathrm{H}), 3.85(\mathrm{dd}, 1 \mathrm{H}$, $J=10.4,2.7 \mathrm{~Hz}), 7.24-7.33(\mathrm{~m}, 3 \mathrm{H}), 7.37-7.40(\mathrm{~m}, 2 \mathrm{H}) ;{ }^{13} \mathrm{C}-\mathrm{NMR}\left(300 \mathrm{MHz}, \mathrm{CDCl}_{3}\right) \delta 12.4$, 20.2, 46.6, 53.6, 60.8, 61.3, 61.9, 127.2, 127.4, 127.8, 128.4, 128.7, 143.0; MS (ESI, $\mathrm{m} / \mathrm{z}$ ): 205.2 $[\mathrm{M}+\mathrm{H}]^{+}$. Anal. Calcd. For $\mathrm{C}_{13} \mathrm{H}_{20} \mathrm{~N}_{2}$ (204.31): C, 76.42; H, 9.87; N, 13.71. Found: C, 76.58; H, 9.90; N, 13.52 .

1-Benzyl-3-phenylpiperazine (5e). This compound was prepared in a similar way to $\mathbf{5 c}$, using compound $4 \mathrm{e}(3.0 \mathrm{~g}, 8.52 \mathrm{~mol})$, as a light yellow solid $(1.93 \mathrm{~g}, 90 \%)$; mp $55-56{ }^{\circ} \mathrm{C}$ (lit, ${ }^{2} 55-63$ $\left.{ }^{\circ} \mathrm{C}\right)$; IR $\left(\mathrm{KBr}, \mathrm{cm}^{-1}\right)$ 3244, 2802, 1602, 761, 741, 701, 696; ${ }^{1} \mathrm{H}-\mathrm{NMR}\left(300 \mathrm{MHz}, \mathrm{CDCl}_{3}\right) \delta 1.99$ (bs, $1 \mathrm{H}), 2.05-2.13(\mathrm{~m}, 1 \mathrm{H}), 2.21-2.26(\mathrm{~m}, 1 \mathrm{H}), 2.84-2.94(\mathrm{~m}, 2 \mathrm{H}), 3.07-3.11(\mathrm{~m}, 2 \mathrm{H}), 3.57(\mathrm{~s}$, $2 \mathrm{H}), 3.91(\mathrm{dd}, 1 \mathrm{H}, J=10.4,2.7 \mathrm{~Hz}), 7.24-7.38(\mathrm{~m}, 10 \mathrm{H}) ;{ }^{13} \mathrm{C}-\mathrm{NMR}\left(300 \mathrm{MHz}, \mathrm{CDCl}_{3}\right) \delta 46.6$, 53.6, 60.8, 61.6, 63.8, 127.5, 127.9, 128.7, 128.8, 129.7, 138.3, 143.0; MS (ESI, $m / z): 253.1$ $[\mathrm{M}+\mathrm{H}]^{+}$. Anal. Calcd. For $\mathrm{C}_{17} \mathrm{H}_{20} \mathrm{~N}_{2}$ (252.35): C, 80.91; H, 7.99; N, 11.10. Found: C, 80.98; H, $7.96 ; \mathrm{N}, 11.06$.

1-Methyl-2-oxo-3-phenylpiperazine (6a). The mixture of 3a (15.0 g, $53.57 \mathrm{mmol}), 5 \% \mathrm{Pd}-\mathrm{C}$ (3.0 g, $50 \%$ wet) and acetic acid $(90 \mathrm{~mL})$ was hydrogenated with $\mathrm{H}_{2}\left(100 \mathrm{psi}\right.$.) at $25-30{ }^{\circ} \mathrm{C}$ for 5 $\mathrm{h}$, filtered and the filtrate was evaporated to dryness. Dissolved the residue in water $(75 \mathrm{~mL}), \mathrm{pH}$ adjusted to 10.5 with $20 \% \mathrm{NaOH}$ and extracted with dichloromethane $(150 \mathrm{~mL})$. Combined organic solution was concentrated to dryness to yield 6a (10.0 g, 98\%) as light yellow oil; IR $\left(\right.$ neat, $\left.\mathrm{cm}^{-1}\right)$ 3301, 2925, 2865, 1640, 1588, 738, 699; ${ }^{1} \mathrm{H}-\mathrm{NMR}\left(300 \mathrm{MHz}, \mathrm{CDCl}_{3}\right) \delta 1.99$ (bs, $1 \mathrm{H}), 3.03(\mathrm{~s}, 3 \mathrm{H}), 3.05-3.19(\mathrm{~m}, 2 \mathrm{H}), 3.31-3.55(\mathrm{~m}, 2 \mathrm{H}), 4.58(\mathrm{~s}, 1 \mathrm{H}), 7.27-7.42(\mathrm{~m}, 5 \mathrm{H}) ;{ }^{13} \mathrm{C}-$ NMR (300 MHz, $\left.\mathrm{CDCl}_{3}\right) \delta 35.1,41.5,50.6,64.2,128.0,128.7,140.3,169.1$; MS (ESI, $\mathrm{m} / \mathrm{z}$ ): 191.0 [M+H] $]^{+}$. Anal. Calcd. For $\mathrm{C}_{11} \mathrm{H}_{14} \mathrm{~N}_{2} \mathrm{O}$ (190.24): C, 69.45; H, 7.42; N, 14.73. Found: C, 69.54; H, 7.35; N, 14.75. 
1-Ethyl-2-oxo-3-phenylpiperazine (6b). This compound was prepared in a similar way to $6 \mathbf{a}$, using compound $\mathbf{3 b}$ (2.5 g, $8.50 \mathrm{mmol}$ ), as a light yellow oil (1.64 g, 95\%); IR (neat, $\left.\mathrm{cm}^{-1}\right) 3312$, 2934, 2873, 1639, 1451, 752, 698; ${ }^{1} \mathrm{H}-\mathrm{NMR}\left(300 \mathrm{MHz}, \mathrm{CDCl}_{3}\right) \delta 1.19$ (t, 3H, J=7.2 Hz), 2.10 (bs, $1 \mathrm{H}), 3.02-3.10(\mathrm{~m}, 1 \mathrm{H}), 3.13-3.20(\mathrm{~m}, 1 \mathrm{H}), 3.27-3.37(\mathrm{~m}, 1 \mathrm{H}), 3.39-3.44(\mathrm{~m}, 1 \mathrm{H}), 3.47-3.58$ $(\mathrm{m}, 2 \mathrm{H}), 4.55(\mathrm{~s}, 1 \mathrm{H}), 7.27-7.40(\mathrm{~m}, 5 \mathrm{H}) ;{ }^{13} \mathrm{C}-\mathrm{NMR}\left(300 \mathrm{MHz}, \mathrm{CDCl}_{3}\right) \delta 12.6,41.5,42.4,47.7$, 64.1, 128.4, 128.6, 128.7, 128.8, 129.3, 140.4, 168.5; MS (ESI, $m / z): 205.2[\mathrm{M}+\mathrm{H}]^{+}$. Anal. Calcd. For $\mathrm{C}_{12} \mathrm{H}_{16} \mathrm{~N}_{2} \mathrm{O}$ (204.27): C, 70.56; H, 7.90; N, 13.71. Found: C, 70.34; H, 7.92; N, 13.74. 1-Allyl-2-oxo-3-phenylpiperazine (6c). This compound was prepared in a similar way to 5c, using compound 3c (2.5 g, $7.91 \mathrm{mmol})$, as a light yellow oil (1.53 g, 90\%); IR (neat, $\left.\mathrm{cm}^{-1}\right) 3309$, 3083, 2865, 1638, 1490, 751, 700; ${ }^{1} \mathrm{H}-\mathrm{NMR}\left(300 \mathrm{MHz}, \mathrm{CDCl}_{3}\right) \delta 1.99$ (bs, 1H), 2.88-2.95 (m, $1 \mathrm{H}), 3.09-3.17(\mathrm{~m}, 1 \mathrm{H}), 3.19-3.22(\mathrm{~m}, 1 \mathrm{H}), 3.32-3.49(\mathrm{~m}, 1 \mathrm{H}), 4.07(\mathrm{~d}, 2 \mathrm{H}, J=6.0 \mathrm{~Hz}), 4.61(\mathrm{~s}$, $1 \mathrm{H})$, 5.20-5.26 (m, 2H), 5.81-5.97 (m, 1H), 7.28-7.40 (m, 5H); ${ }^{13} \mathrm{C}-\mathrm{NMR}\left(300 \mathrm{MHz}, \mathrm{CDCl}_{3}\right) \delta$ 41.6, 47.9, 49.7, 64.3, 118.2, 128.0, 128.7, 132.9, 140.3, 168.8; MS (ESI, $m / z): 217.2[\mathrm{M}+\mathrm{H}]^{+}$. Anal. Calcd. For $\mathrm{C}_{13} \mathrm{H}_{16} \mathrm{~N}_{2} \mathrm{O}$ (216.28): C, 72.19; H, 7.46; N, 12.95. Found: C, 72.06; H, 7.58; N, 12.98 .

1-Benzyl-2-oxo-3-phenylpiperazine (6d). This compound was prepared in a similar way to 5c, using compound $3 \mathrm{e}(2.5 \mathrm{~g}, 6.83 \mathrm{mmol})$, as a light yellow oil (1.59 g, 88\%); IR (neat, $\left.\mathrm{cm}^{-1}\right) 3313$, 2862, 1645, 753, 700; ${ }^{1} \mathrm{H}-\mathrm{NMR}\left(300 \mathrm{MHz}, \mathrm{CDCl}_{3}\right) \delta 1.91(\mathrm{bs}, 1 \mathrm{H}), 2.96-3.00(\mathrm{~m}, 1 \mathrm{H}), 3.20-3.24$ $(\mathrm{m}, 1 \mathrm{H}), 3.42-3.46(\mathrm{~m}, 1 \mathrm{H}), 3.69-3.72(\mathrm{~m}, 1 \mathrm{H}), 4.67(\mathrm{~s}, 2 \mathrm{H}), 5.31(\mathrm{~s}, 1 \mathrm{H}), 7.20-7.49(\mathrm{~m}, 10 \mathrm{H})$; ${ }^{13} \mathrm{C}-\mathrm{NMR}\left(300 \mathrm{MHz}, \mathrm{CDCl}_{3}\right) \delta 41.6,47.5,50.7,64.2,127.9,128.0,128.4,128.6,128.7,128.9$, 129.0, 129.1, 129.3, 129.4, 137.1, 139.7, 168.7; MS (ESI, $m / z): 267.1[\mathrm{M}+\mathrm{H}]^{+}$. Anal. Calcd. For $\mathrm{C}_{17} \mathrm{H}_{18} \mathrm{~N}_{2} \mathrm{O}$ (266.34): C, 76.66; H, 6.81; N, 10.52. Found: C, 76.50; H, 6.83; N, 10.54.

General method for the preparation of 1-alky-3-phenylpiperazine (5a-c, e) by the reduction of 1-alkyl-2-oxo-3-phenylpiperazines (6a-d). 1-Methyl-3-phenylpiperazine (5a). Under $\mathrm{N}_{2}$ atmosphere, 6a $(9.0 \mathrm{~g}, 47.36 \mathrm{mmol})$ was added slowly to a suspension of $\mathrm{LiAlH}_{4}(3.1 \mathrm{~g}$, $81.58 \mathrm{mmol})$ in anhydrous THF $(100 \mathrm{~mL})$ at $10-15^{\circ} \mathrm{C}$ over a period of $1 \mathrm{~h}$ and then refluxed for 6 h. The excess $\mathrm{LiAlH}_{4}$ was destroyed at $0{ }^{\circ} \mathrm{C}$ with water $(3.1 \mathrm{~mL}), 15 \% \mathrm{NaOH}(3.1 \mathrm{~mL})$ and water $(9.3 \mathrm{~mL})$. After filtration, the filtrate was evaporated to dryness and dissolved the residue in water $(25 \mathrm{~mL})$. Acidified the solution to $\mathrm{pH} 2.0$ with $15 \% \mathrm{HCl}$ and washed the solution with toluene $(20 \mathrm{~mL})$. Basified to $\mathrm{pH} 10.5$ with $20 \% \mathrm{NaOH}$. The aqueous solution was extracted with dichloromethane $(100 \mathrm{~mL})$; the combined organic solution was evaporated. The residue was treated with cyclohexane $(20 \mathrm{~mL})$ to yield $\mathbf{5 a}(7.4 \mathrm{~g}, 84 \%)$ as a light yellow solid; purity $99.5 \%$ (by HPLC), mp 58-59 ${ }^{\circ} \mathrm{C}$; ${ }^{1} \mathrm{H}-\mathrm{NMR}\left(300 \mathrm{MHz}, \mathrm{CDCl}_{3}\right) \delta 1,80(\mathrm{bs}, 1 \mathrm{H}), 1.96-2.03(\mathrm{~m}, 1 \mathrm{H}), 2.13-$ $2.18(\mathrm{~m}, 1 \mathrm{H}), 2.31(\mathrm{~s}, 3 \mathrm{H}), 2.80-2.89(\mathrm{~m}, 2 \mathrm{H}), 3.06-3.10(\mathrm{~m}, 2 \mathrm{H}), 3.86(\mathrm{dd}, 1 \mathrm{H}, J=10.6,2.7 \mathrm{~Hz})$, 7.23-7.40 (m, 5H); MS (ESI, $m / z): 177.0[\mathrm{M}+\mathrm{H}]^{+}$. Similarly, by following the same procedure $\mathbf{5 b}, \mathbf{5 c}$ and $5 \mathrm{e}$ can be prepared. 


\section{Acknowledgements}

Authors thank the management of Aurobindo Pharma Limited, Hyderabad for permission to publish this work. Authors also thank the Analytical Research Department for their valuable contribution to this work.

\section{References and Footnotes}

1. Van der Burg, W. J. Ger. Offen. 2, 614, 406, 1975; Chem. Abstr. 1977, 86, 29883.

2. Roderick, W. R.; Platte, H. J.; Pollard, C. B. J. Med. Chem. 1966, 9, 181-185.

3. Dolitzky, Ben-Zion. PCT Int. Appl. WO 00/63, 185: Chem. Abstr. 2000, 133, 321901.

4. Bosch i Llado, J.; Camps Garcia, P.; Contreras Lascorz, J.; Onrubia Miguel, M. PCT Int. Appl. WO 03/024, 918; Chem. Abstr. 2003, 138, 254962.

5. Guo, B. S.; Yang, Y. S.; Ji, R. Y. Chinese Chemical Lett. 2003, 14, 365.

6. Ramamohan Rao, D.; Sankara Rao, Ch.; Sreenivasulu, P. PCT Int. Appl. WO 02 090, 339, 2002; Chem. Abstr. 2002, 137, 370108.

7. Handa, V. K.; Srinivasarao D. V. N.; Sivakumaran, M. US. Pat. Appl. 242, 879, 2004 : Chem. Abstr. 2005, 142, 23309.

8. HPLC analyses were carried out only for compounds $2 \mathrm{a}, 3 \mathrm{a}, 4 \mathrm{a}$ and $5 \mathrm{a}$. Retention times were $12.1,13.1,10.3$ and 6.5 respectively $\left(\mathrm{CH}_{3} \mathrm{CN}\right.$ : $\left.0.05 \mathrm{M} \mathrm{KH}_{2} \mathrm{PO}_{4}\right)$.

9. Lithium aluminum hydride reacts violently with water, liberating hydrogen, incompatible with strong oxidizing agents. Reactions to be carried out in anhydrous conditions.

10. Palladium on carbon is flammable, pyrophoric after activation with hydrogen. It should always be kept at inert atmosphere. 